Pacific

Journal of

Mathematics

KNOTS YIELDING HOMEOMORPHIC LENS SPACES BY DEHN SURGERY

Toshio SAito And MASAKazU Teragaito

Volume 244 No. 1

January 2010 


\title{
KNOTS YIELDING HOMEOMORPHIC LENS SPACES BY DEHN SURGERY
}

\author{
Toshio SAito And MAsakazu TERAGAito
}

\begin{abstract}
We show that there exist infinitely many pairs of distinct knots in the 3sphere such that each pair can yield homeomorphic lens spaces by the same Dehn surgery. Moreover, each knot of the pair can be chosen to be a torus knot, a satellite knot or a hyperbolic knot, except that both cannot be satellite knots simultaneously. This exception is shown to be unavoidable by the classical theory of binary quadratic forms.
\end{abstract}

\section{Introduction}

For a knot $K$ in the 3-sphere $S^{3}$, let $K(\mathrm{~m} / \mathrm{n})$ denote the closed oriented 3-manifold obtained by $m / n$-Dehn surgery on $K$, that is, $K(m / n)$ is the union of the knot exterior $E(K)=S^{3}-\operatorname{int} N(K)$ and a solid torus $V$ in such a way that the meridian of $V$ is attached to a loop on $\partial E(K)$ with slope $m / n$. In this paper, all 3-manifolds are oriented, and two knots in $S^{3}$ are said to be equivalent if there is an orientationpreserving homeomorphism of $S^{3}$ sending one to the other.

For a fixed slope $m / n, m / n$-surgery can be regarded as a map from the set of the equivalence classes of knots to that of 3-manifolds. There are many results on the injectivity of this map. Lickorish [1976] gave two nonequivalent knots on which $(-1)$-surgeries yield the same homology sphere. Brakes [1980] showed that for any integer $n \geq 2$, there exist $n$ distinct knots on which 1-surgeries yield the same 3-manifold. See also [Kawauchi 1996; Livingston 1982; Teragaito 1994]. Finally, Osoinach [1998; 2006] showed the existence of 3-manifolds, in fact, a hyperbolic 3-manifold and a toroidal manifold, which can be obtained from infinitely many hyperbolic knots by 0-surgery. By using Osoinach's construction, Teragaito [2007] gave a Seifert fibered manifold over the 2-sphere with three exceptional fibers that can be obtained from infinitely many hyperbolic knots by 4 -surgery. Thus it is natural to ask whether there exists a lens space that can be obtained from infinitely

MSC2000: primary 57M25; secondary 11B39, 11E16.

Keywords: Dehn surgery, lens space, knot, Fibonacci number, binary quadratic form.

Saito was partially supported by the Japan Society for the Promotion of Science, Research Fellowships for Young Scientists. Teragaito was partially supported by the Japan Society for the Promotion of Science, Grant-in-Aid for Scientific Research (C), 19540089. 
many knots by the same Dehn surgery. Although we do not know the answer yet, we feel that it is negative based on our computer experiments. In fact, as far as we know, at most two knots can yield homeomorphic lens spaces by the same Dehn surgery.

We should note that Berge's table [1990s] shows that among the lens spaces with fundamental groups of order up to 500, there are 32 that admit two knots yielding $S^{3}$ by Dehn surgery. This strongly suggests that many lens spaces can be obtained from nonequivalent knots in $S^{3}$ by the same Dehn surgery. In this paper, we study whether a pair of nonequivalent knots can yield homeomorphic lens spaces, ignoring orientations, by the same Dehn surgery. We should be attentive to this orientation convention. Let $U$ be the unknot and $K$ a knot in $S^{3}$. By using Floer homology for Seiberg-Witten monopoles, it is proved in [Kronheimer et al. 2007] that if there exists an orientation-preserving homeomorphism between $K(m / n)$ and $U(m / n)$, then $K$ is trivial. In other words, if $K(m / n)$ is homeomorphic to the lens space $L(m, n)$ under an orientation-preserving homeomorphism, then $K$ is trivial. Here, the preservation of orientation is important, because 5-surgery on the right-handed trefoil yields $L(5,4)=L(5,-1)$. From our point of view, the right-handed trefoil and the unknot yield homeomorphic lens spaces under the same 5-surgery.

As a consequence of the cyclic surgery theorem [Culler et al. 1987], any nontrivial amphicheiral knot has no Dehn surgery yielding a lens space, and the pair of a knot and its mirror image cannot yield homeomorphic lens spaces by the same Dehn surgery. Also, only torus knots admit nonintegral lens space surgeries.

Our first result is the following. We recall that all knots are classified into three families: torus knots, satellite knots, and hyperbolic knots.

Theorem 1.1. There exist infinitely many pairs $\left\{K_{1}, K_{2}\right\}$ of nonequivalent knots in $S^{3}$ such that $m$-surgeries on them yield homeomorphic lens spaces for some integer $m$. Also, $K_{i}$ can be chosen to be a torus knot, a satellite knot or a hyperbolic knot, except that $K_{1}$ and $K_{2}$ cannot be satellite knots simultaneously.

The exceptional case in Theorem 1.1 is unavoidable as shown in Corollary 1.3, which is obtained as a consequence of the next theorem.

Theorem 1.2. (1) There exist infinitely many pairs of nonequivalent torus knots in $S^{3}$ such that some half-integral surgeries on them yield homeomorphic lens spaces.

(2) Let $K_{1}$ and $K_{2}$ be nonequivalent torus knots. Suppose a slope $r$ corresponds to a lens space surgery for both $K_{1}$ and $K_{2}$. If the slope $r$ runs at least three times in the longitudinal direction, then $r$-surgeries on $K_{1}$ and $K_{2}$ cannot yield homeomorphic lens spaces. 
Corollary 1.3. Nonequivalent satellite knots cannot yield homeomorphic lens spaces by the same Dehn surgery.

Question 1.4. Is there a lens space that can be obtained from three nonequivalent knots in $S^{3}$ by the same Dehn surgery?

Based on a computer experiment, we conjecture that the answer is negative.

The paper is organized as follows. In Section 2, we give infinitely many pairs of torus knots that yield homeomorphic lens spaces. After establishing one result concerning a divisibility of integers by using the classical theory of integral binary quadratic forms in Section 3, we prove Theorem 1.2 and Corollary 1.3 in Section 4. In Section 5, we review one special class of doubly primitive knots. In Section 6, we construct by using tangles infinitely many pairs of hyperbolic knots that yield homeomorphic lens spaces. Finally, Section 7 completes the proof of Theorem 1.1 by treating the case where the knots of a pair belong to different classes.

\section{Torus knots}

Here, we give infinitely many pairs of torus knots that yield homeomorphic lens spaces by the same integral Dehn surgery.

Recall that the Fibonacci numbers are defined by the recurrence equation

$$
F_{n+2}=F_{n+1}+F_{n} \quad \text { with } F_{0}=0 \text { and } F_{1}=1 .
$$

We make use of Cassini's identity (see [Graham et al. 1994])

$$
F_{k-1} F_{k+1}-F_{k}^{2}=(-1)^{k} \text { for } k>0 .
$$

Let $a_{n}=F_{n+2}$ and $b_{n}=F_{n+3}+F_{n+1}$ for $n \geq 1$.

Lemma 2.1. For any $n \geq 1$,

$$
a_{n+1} b_{n}+(-1)^{n+1}=a_{n} b_{n+1}+(-1)^{n} .
$$

Proof. By using Cassini's identity,

$$
\begin{aligned}
a_{n+1} b_{n}+(-1)^{n+1} & =F_{n+3}\left(F_{n+3}+F_{n+1}\right)+(-1)^{n+1} \\
& =F_{n+3}^{2}+F_{n+3} F_{n+1}+(-1)^{n+1}=F_{n+3}^{2}+F_{n+2}^{2} .
\end{aligned}
$$

Similarly,

$$
\begin{aligned}
a_{n} b_{n+1}+(-1)^{n} & =F_{n+2}\left(F_{n+4}+F_{n+2}\right)+(-1)^{n} \\
& =F_{n+2} F_{n+4}+F_{n+2}^{2}+(-1)^{n}=F_{n+3}^{2}+F_{n+2}^{2} .
\end{aligned}
$$

As seen from Cassini's identity, two successive Fibonacci numbers are relatively prime. Then it is easy to see that $\operatorname{gcd}\left(a_{n+1}, b_{n}\right)=\operatorname{gcd}\left(a_{n}, b_{n+1}\right)=1$. 
Proposition 2.2. For $n \geq 1$, let $K$ be the torus knot of type $\left(a_{n+1}, b_{n}\right)$, and $K^{\prime}$ the torus knot of type $\left(a_{n}, b_{n+1}\right)$. Let $m=a_{n+1} b_{n}+(-1)^{n+1}\left(=a_{n} b_{n+1}+(-1)^{n}\right)$. Then $K$ and $K^{\prime}$ are not equivalent, and $m$-surgery on $K$ and $K^{\prime}$ yields homeomorphic lens spaces.

Proof. $K$ and $K^{\prime}$ are not equivalent since $a_{n}<a_{n+1}<b_{n}<b_{n+1}$. By [Moser 1971], $m$-surgery on $K$ and $K^{\prime}$ yields the lens spaces $L\left(a_{n+1} b_{n}+(-1)^{n+1}, a_{n+1}^{2}\right)$ and $L\left(a_{n} b_{n+1}+(-1)^{n}, a_{n}^{2}\right)$, respectively. Since $a_{n}^{2}+a_{n+1}^{2}=F_{n+2}^{2}+F_{n+3}^{2}=m$ as seen in the proof of Lemma $2.1, a_{n}^{2}+a_{n+1}^{2} \equiv 0(\bmod m)$. Thus these lens spaces are homeomorphic.

\section{Binary quadratic form}

In this section, we prove Proposition 3.1, which will be used in Section 4. For its proof, we quickly review the classical theory of integral binary quadratic forms. See [Flath 1989], for example.

Let $f(x, y)=A x^{2}+B x y+C y^{2}$ be an integral binary quadratic form with discriminant $\Delta=B^{2}-4 A C$. For our purposes, it is enough to assume that $\Delta$ is a positive nonsquare. Let $m$ be a nonzero integer. Then there is a finite algorithm to find all integral solutions $(x, y) \in \mathbb{Z}^{2}$ of $f(x, y)=m$, as described below.

Let $\mathscr{Y}=\left\{(x, y) \in \mathbb{Z}^{2} \mid f(x, y)=m\right\}$ be the set of integral solutions of $f(x, y)=m$. Set

$$
\rho= \begin{cases}\frac{1}{2} \sqrt{\Delta} & \text { if } \Delta \equiv 0(\bmod 4), \\ \frac{1}{2}(1+\sqrt{\Delta}) & \text { if } \Delta \equiv 1(\bmod 4) .\end{cases}
$$

Let us consider the ring $O_{\Delta}=\{x+y \rho \mid x, y \in \mathbb{Z}\}$. Let $O_{\Delta}^{\times}$be the group of units of $\mathrm{O}_{\Delta}$, and let $\mathrm{O}_{\Delta, 1}^{\times}=\left\{\alpha \in \mathcal{O}_{\Delta}^{\times} \mid N(\alpha)=1\right\}$ be the subgroup of units for norm 1 . Note that the norm $N(\alpha)$ of $\alpha=x+y \rho$ is given

$$
N(\alpha)= \begin{cases}x^{2}-\frac{1}{4} \Delta y^{2} & \text { if } \Delta \equiv 0(\bmod 4), \\ x^{2}+x y-\frac{1}{4}(\Delta-1) y^{2} & \text { if } \Delta \equiv 1(\bmod 4) .\end{cases}
$$

In fact, $\mathbb{O}_{\Delta, 1}^{\times}$corresponds to the solution set of the Pell equation $N(\alpha)=1$. Then $\mathcal{O}_{\Delta, 1}^{\times}$acts on the set $\mathscr{Y}$. It is well known that the number of $\mathcal{O}_{\Delta, 1}^{\times}$-orbits in $\mathscr{Y}$ is finite. Since $0_{\Delta, 1}^{\times}$is infinite, the orbit of each solution is infinite, so $\mathscr{Y}$ is infinite unless $\mathscr{Y}=\varnothing$. The action is explicitly given by the formulas

$$
\left(x^{\prime}, y^{\prime}\right)=\left\{\begin{array}{cc}
(x, y)\left(\begin{array}{cc}
u-\frac{1}{2} B v & A v \\
-C v & u+\frac{1}{2} B v
\end{array}\right) & \text { if } \Delta \equiv 0(\bmod 4), \\
(x, y)\left(\begin{array}{cc}
u+\frac{1}{2}(1-B) v & A v \\
-C v & u+\frac{1}{2}(1+B) v
\end{array}\right) & \text { if } \Delta \equiv 1(\bmod 4),
\end{array}\right.
$$

for $u+v \rho \in \mathbb{O}_{\Delta, 1}^{\times}$and $(x, y) \in \mathscr{Y}$. 
Let $\tau$ be the smallest unit of $\mathbb{O}_{\Delta, 1}^{\times}$that is greater than 1 . Then every $\mathbb{O}_{\Delta, 1}^{\times}$-orbit of integral solutions of $f(x, y)=m$ contains a solution $(x, y) \in \mathbb{Z}^{2}$ such that

$$
0 \leq y \leq U= \begin{cases}|(A m / \Delta)(\tau+\bar{\tau}-2)|^{1 / 2} & \text { if } A m>0, \\ |(A m / \Delta)(\tau+\bar{\tau}+2)|^{1 / 2} & \text { if } A m<0,\end{cases}
$$

where $\bar{\tau}$ is the conjugate of $\tau$. Also, two distinct solutions $\left(x_{1}, y_{1}\right),\left(x_{2}, y_{2}\right) \in \mathbb{Z}^{2}$ of $f(x, y)=m$ such that $0 \leq y_{i} \leq U$ belong to the same $\mathbb{O}_{\Delta, 1}^{\times}$-orbit if and only if $y_{1}=y_{2}=0$ or $y_{1}=y_{2}=U$.

Proposition 3.1. Let $n \geq 3$ be an integer. Let $a, b$ and $c$ be positive integers such that $a>1$ and $\operatorname{gcd}(a, b)=\operatorname{gcd}(a, c)=1$. Then $b^{2} \pm c^{2}$ is not divisible by $n a b c \pm 1$.

Proof. Without loss of generality, we may assume that $b>c$. Let $\varepsilon \in\{1,-1\}$. If $b^{2}+c^{2}$ is divisible by $n a b c+\varepsilon$, then

$$
b^{2}+c^{2}=Q(n a b c+\varepsilon)
$$

for some integer $Q \geq 1$. Consider an integral binary quadratic form $f(x, y)=$ $x^{2}-Q$ naxy $+y^{2}$. Then Equation (3-1) means that the equation $f(x, y)=\varepsilon Q$ has a solution $(b, c)$.

Similarly, if $b^{2}-c^{2}$ is divisible by $n a b c+\varepsilon$, then for a binary quadratic form $g(x, y)=x^{2}-Q$ naxy $-y^{2}$, the equation $g(x, y)=\varepsilon Q$ has a solution $(b, c)$. We remark that the discriminants $\Delta_{f}=(Q n a)^{2}-4$ of $f$ and $\Delta_{g}=(Q n a)^{2}+4$ of $g$ are positive and nonsquare.

First, we list all solutions in positive integers of the equation $f(x, y)=Q$. For simplicity, let $\Delta=\Delta_{f}$. Let $\mathscr{S}=\left\{(x, y) \in \mathbb{Z}^{2} \mid f(x, y)=Q\right\}$ be the set of all integral solutions of the equation $f(x, y)=Q$. Then the action of $0_{\Delta, 1}^{\times}$on the set $\mathscr{Y}$ is given by the formula

$$
\left(x^{\prime}, y^{\prime}\right)=\left\{\begin{array}{cc}
(x, y)\left(\begin{array}{cc}
u+\frac{1}{2} Q n a v & v \\
-v & u-\frac{1}{2} Q n a v
\end{array}\right) & \text { if } \Delta \equiv 0(\bmod 4), \\
(x, y)\left(\begin{array}{cc}
u+\frac{1}{2}(1+Q n a) v & v \\
-v & u+\frac{1}{2}(1-Q n a) v
\end{array}\right) & \text { if } \Delta \equiv 1(\bmod 4),
\end{array}\right.
$$

for $u+v \rho \in \mathbb{O}_{\Delta, 1}^{\times}$and $(x, y) \in \mathscr{Y}$.

Let $\tau$ be the smallest unit of $O_{\Delta, 1}^{\times}$that is greater than 1 . In fact, we see that

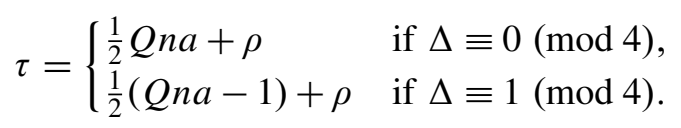

Then every orbit contains a solution $(x, y) \in \mathbb{Z}^{2}$ such that

$$
0 \leq y \leq U=|(Q / \Delta)(\tau+\bar{\tau}-2)|^{1 / 2} .
$$


In our case, $U<1$, and so $\mathscr{S}$ consists of a single $\mathbb{O}_{\Delta, 1}^{\times}$-orbit. Furthermore, $Q$ must be a square in order that $\mathscr{Y} \neq \varnothing$. We start a solution $(\sqrt{Q}, 0) \in \mathscr{Y}$. By (3-2),

$$
\tau \cdot(\sqrt{Q}, 0)=(\sqrt{Q}, 0)\left(\begin{array}{cc}
Q n a & 1 \\
-1 & 0
\end{array}\right)=\left(Q^{3 / 2} n a, \sqrt{Q}\right) .
$$

Since

$$
(x, y)\left(\begin{array}{cc}
\text { Qna } & 1 \\
-1 & 0
\end{array}\right)=(\text { Qnax }-y, x),
$$

every solution in positive integers has a coordinate that is divisible by $a$. Thus $f(x, y)=Q$ cannot have the solution $(b, c)$, because $\operatorname{gcd}(a, b)=\operatorname{gcd}(a, c)=1$.

For the equation $f(x, y)=-Q$, we have $U=|(-Q / \Delta)(\tau+\bar{\tau}+2)|^{1 / 2}<1$ again. However, $f(x, 0)=x^{2}$ implies that the set of solutions of the equation $f(x, y)=-Q$ is empty.

Next, consider the equation $g(x, y)=Q$. Let $\mathscr{T}=\left\{(x, y) \in \mathbb{Z}^{2} \mid g(x, y)=Q\right\}$. Put $\Delta=\Delta_{g}$. Then $\mathrm{O}_{\Delta}, \mathrm{O}_{\Delta, 1}^{\times}$are defined in the same way, but the action of $\mathrm{O}_{\Delta, 1}^{\times}$ on the set $\mathscr{T}$ is given by the formula

$$
\left(x^{\prime}, y^{\prime}\right)= \begin{cases}(x, y)\left(\begin{array}{cc}
u+\frac{1}{2} Q n a v & v \\
v & u-\frac{1}{2} Q n a v
\end{array}\right) & \text { if } \Delta \equiv 0(\bmod 4), \\
(x, y)\left(\begin{array}{cc}
u+\frac{1}{2}(1+Q n a) v & v \\
v & u+\frac{1}{2}(1-Q n a) v
\end{array}\right) & \text { if } \Delta \equiv 1(\bmod 4),\end{cases}
$$

for $u+v \rho \in \mathcal{O}_{\Delta, 1}^{\times}$and $(x, y) \in \mathscr{T}$. Also, the smallest unit $\tau$ of $\mathcal{O}_{\Delta, 1}^{\times}$that is greater than 1 is given by

$$
\tau= \begin{cases}\left(\frac{1}{2} Q n a+\rho\right)^{2}=\frac{1}{2}(Q n a)^{2}+1+Q n a \rho & \text { if } \Delta \equiv 0(\bmod 4), \\ \left(\frac{1}{2}(Q n a-1)+\rho\right)^{2}=\frac{1}{2}\left((Q n a)^{2}-Q n a\right)+1+Q n a \rho & \text { if } \Delta \equiv 1(\bmod 4) .\end{cases}
$$

As before, we can evaluate $U=|(Q / \Delta)(\tau+\bar{\tau}-2)|^{1 / 2}<\sqrt{Q}$.

On the other hand, if $(x, y) \in \mathcal{T}$, then $\Delta y^{2}+4 Q=(2 x-\text { Qnay })^{2}$. That is, $\Delta y^{2}+4 Q$ must be a square. If $0<y<\sqrt{Q}$, then

$$
\text { Qnay }<\sqrt{\Delta y^{2}+4 Q}<\text { Qnay }+1 .
$$

Hence $y=0$, and so $\mathscr{T}$ consists of a single $\mathcal{O}_{\Delta, 1}^{\times}$-orbit. Thus $Q$ must be a square in order that $\mathscr{T} \neq \varnothing$. Starting a solution $(\sqrt{Q}, 0) \in \mathscr{T}$, we have

$$
\tau \cdot(\sqrt{Q}, 0)=(\sqrt{Q}, 0)\left\{\begin{array}{cc}
(Q n a)^{2}+1 & Q n a \\
Q n a & 1
\end{array}\right\}=\left(Q^{5 / 2} n^{2} a^{2}+\sqrt{Q}, Q^{3 / 2} n a\right)
$$

by the formulas (3-3). Thus for every solution in positive integers, the second coordinate is divisible by $a$. 
Finally, for the equation $g(x, y)=-Q$, we have $U=|(-Q / \Delta)(\tau+\bar{\tau}+2)|^{1 / 2}$, which is less than or equal to $\sqrt{Q}$ when $\Delta \equiv 0(\bmod 4)$ and less than $\sqrt{Q}$ when $\Delta \equiv 1(\bmod 4)$.

If $g(x, y)=-Q$, then $\Delta y^{2}-4 Q=(2 x-Q n a y)^{2}$. Thus $y \neq 0$. Furthermore, if $y<\sqrt{Q}$, then

$$
\text { Qnay }-1<\sqrt{\Delta y^{2}-4 Q}<\text { Qnay. }
$$

Therefore, $y=\sqrt{Q}$ is the only possibility, and so $Q$ must be a square. As before, the set of solutions of the equation $g(x, y)=-Q$ consists of a single $\mathbb{O}_{\Delta, 1}^{\times}$-orbit, whose representative is $(0, \sqrt{Q})$. Then

$$
\tau \cdot(0, \sqrt{Q})=(0, \sqrt{Q})\left(\begin{array}{cc}
(Q n a)^{2}+1 & Q n a \\
Q n a & 1
\end{array}\right)=\left(Q^{3 / 2} n a, \sqrt{Q}\right) .
$$

Hence the first coordinate is divisible by $a$ for any solution in positive integers.

Remark 3.2. The requirement $a>1$ in Proposition 3.1 is necessary. For example, let $a=1, b=3, c=8$ and $n=3$. Then $b^{2}+c^{2}=73$ is divisible by $n a b c+1=73$.

\section{Nonintegral surgery on torus knots}

In this section, we prove Theorem 1.2.

Let $\left\{a_{n}\right\}$ and $\left\{b_{n}\right\}$ be the sequences of positive integers defined by

$$
a_{n+1}=a_{n}+b_{n} \quad \text { and } \quad b_{n+1}=a_{n+1}+a_{n}
$$

with $a_{1}=2$ and $b_{1}=3$.

Lemma 4.1. For any $n \geq 1$,

(1) $2 a_{n} b_{n+1}+(-1)^{n+1}=2 a_{n+1} b_{n}+(-1)^{n}$,

$$
4 a_{n+1}^{2} b_{n+1}^{2}+1=\left(2 a_{n+1} b_{n+2}+(-1)^{n+2}\right)\left(2 a_{n} b_{n+1}+(-1)^{n+1}\right) .
$$

Proof. By (4-1), we have $a_{n+1}=2 a_{n}+a_{n-1}$. Then

$$
\begin{aligned}
2 a_{n} b_{n+1}-2 a_{n+1} b_{n} & =2 a_{n}\left(a_{n+1}+a_{n}\right)-2 a_{n+1}\left(a_{n+1}-a_{n}\right) \\
& =2\left(a_{n}^{2}-a_{n+1}^{2}+2 a_{n} a_{n+1}\right) \\
& =-2\left(a_{n-1}^{2}-a_{n}+2 a_{n-1} a_{n}\right) \\
& \vdots \\
& =(-1)^{n-1} 2\left(a_{1}^{2}-a_{2}^{2}+2 a_{1} a_{2}\right)=(-1)^{n} 2=(-1)^{n}-(-1)^{n+1} .
\end{aligned}
$$

This proves (1).

To prove (2), we observe that $2 b_{n+1}=a_{n+2}+a_{n}$ by (4-1). Also, as shown above, $2 a_{n} b_{n+1}-2 a_{n+1} b_{n}=(-1)^{n} 2$. Thus, $a_{n} b_{n+1}-a_{n+1} b_{n}=(-1)^{n}$. From (4-1), 
$a_{n}\left(a_{n+1}+a_{n}\right)-a_{n+1}\left(a_{n+1}-a_{n}\right)=(-1)^{n}$. Then $a_{n}^{2}+2 a_{n} a_{n+1}-a_{n+1}^{2}=(-1)^{n}$. Thus,

$$
\begin{aligned}
& \left(2 a_{n+1} b_{n+2}+(-1)^{n+2}\right)\left(2 a_{n} b_{n+1}+(-1)^{n+1}\right) \\
= & \left(2 a_{n+2} b_{n+1}+(-1)^{n+1}\right)\left(2 a_{n} b_{n+1}+(-1)^{n+1}\right) \\
= & 4 a_{n} a_{n+2} b_{n+1}^{2}+(-1)^{n+1} 2 b_{n+1}\left(a_{n}+a_{n+2}\right)+1 \\
= & 4 b_{n+1}^{2}\left(a_{n} a_{n+2}+(-1)^{n+1}\right)+1 \\
= & 4 b_{n+1}^{2}\left(a_{n}\left(a_{n+1}+b_{n+1}\right)+(-1)^{n+1}\right)+1 \\
= & 4 b_{n+1}^{2}\left(a_{n} a_{n+1}+a_{n}\left(a_{n+1}+a_{n}\right)+(-1)^{n+1}\right)+1 \\
= & 4 b_{n+1}^{2}\left(2 a_{n} a_{n+1}+a_{n}^{2}+(-1)^{n+1}\right)+1=4 b_{n+1}^{2} a_{n+1}^{2}+1 .
\end{aligned}
$$

From (1), we have that $\operatorname{gcd}\left(a_{n}, b_{n+1}\right)=\operatorname{gcd}\left(b_{n}, a_{n+1}\right)=1$.

Proof of Theorem 1.2(1). Let $K_{1}$ be the torus knot of type $\left(a_{n}, b_{n+1}\right)$, and let $K_{2}$ be the torus knot of type $\left(b_{n}, a_{n+1}\right)$. Since $a_{n}<b_{n}<a_{n+1}<b_{n+1}$ for any $n \geq 1$, $K_{1}$ and $K_{2}$ are not equivalent. Then $\frac{1}{2}\left(2 a_{n} b_{n+1}+(-1)^{n+1}\right)$-surgery on $K_{1}$ and $\frac{1}{2}\left(2 a_{n+1} b_{n}+(-1)^{n}\right)$-surgery on $K_{2}$ yield the lens spaces

$$
L\left(2 a_{n} b_{n+1}+(-1)^{n+1}, 2 b_{n+1}^{2}\right) \quad \text { and } L\left(2 a_{n+1} b_{n}+(-1)^{n}, 2 a_{n+1}^{2}\right),
$$

respectively. By Lemma 4.1, the surgery coefficients are the same, and the two lens spaces are homeomorphic.

In the rest of this section, we prove Theorem 1.2(2) and Corollary 1.3.

Let $K_{1}$ be the torus knot of type $(p, q)$; let $K_{2}$ be the torus knot of type $(r, s)$. Suppose $n \geq 3$. If $m / n$-surgery on $K_{1}$ yields a lens space, then $\Delta(p q / 1, m / n)=$ $|n p q-m|=1$, so $m=n p q \pm 1$. Hence if $m / n$-surgery on $K_{1}$ and $K_{2}$ yields homeomorphic lens spaces, then $n p q+\varepsilon=n r s+\varepsilon^{\prime}$ for some $\varepsilon, \varepsilon^{\prime} \in\{1,-1\}$. Since we consider nontrivial torus knots, we can assume that $p, q, r$ and $s$ are positive by taking mirror images, if necessary. Moreover, we may assume that $2 \leq q<p, 2 \leq s<r$ and $r<p$. Now $\varepsilon=\varepsilon^{\prime}$ because $n \geq 3$, and so $p q=r s$. By [Moser 1971], $m / n$-surgery on $K_{1}$ and $K_{2}$ yields $L\left(m, n q^{2}\right)$ and $L\left(m, n s^{2}\right)$, respectively.

Theorem 1.2(2) follows directly from the following.

Proposition 4.2. The two lens spaces $L\left(m, n q^{2}\right)$ and $L\left(m, n s^{2}\right)$ are not homeomorphic.

Proof. The two lens spaces are homeomorphic if and only if

$$
\begin{aligned}
n q^{2} & \equiv \pm n s^{2} & & (\bmod m) \text { or } \\
n^{2} q^{2} s^{2} & \equiv \pm 1 & & (\bmod m) .
\end{aligned}
$$

Since $n p q+\varepsilon=n r s+\varepsilon$, we have $n p q=n r s$. Thus $q<s<r<p$. 
First, $n q^{2} \not \equiv n s^{2}(\bmod m)$ because $0<n\left(s^{2}-q^{2}\right)<n s^{2}<n r s-1 \leq m$. Since $n q^{2}+n s^{2}<n(p q+r s)-2=2 n p q-2 \leq 2 m$, the equation $n q^{2} \equiv-n s^{2}(\bmod m)$ is possible only when $n q^{2}+n s^{2}=m$. However, this is impossible because $m$ is not divisible by $n$. The impossibility of Equation (4-3) is shown in Proposition 4.3.

Proposition 4.3. $n^{2} q^{2} s^{2} \not \equiv \pm 1(\bmod m)$.

Proof. Suppose $n^{2} q^{2} s^{2} \equiv 1(\bmod m)$. Then $n^{2} q^{2} s^{2}-1=k m$ for some integer $k \geq 1$. Recall that $m=n p q+\varepsilon$, so $-1 \equiv k \varepsilon(\bmod n)$ and thus $k \equiv-\varepsilon(\bmod n)$. Put $k=n \ell-\varepsilon$ with $\ell \geq 1$. (If $\ell=0$, then $k=-\varepsilon=-1$, so $n^{2} q^{2} s^{2}-1=m$. This implies that $q$ divides $p$, a contradiction.) Then $n^{2} q^{2} s^{2}-1=(n \ell-\varepsilon)(n p q+\varepsilon)$ implies

$$
q\left(n q s^{2}-p(n \ell-\varepsilon)\right)=\varepsilon \ell .
$$

Thus $q$ divides $\ell$, and $\operatorname{gcd}(p, s)$ divides $\ell / q$. For simplicity, we denote $\operatorname{gcd}(x, y)$ by $(x, y)$.

Hence

$$
\begin{aligned}
p(n \ell-\varepsilon) & =n q s^{2}-\varepsilon \ell / q=n q(p, s)^{2}(q, s)^{2}-\varepsilon \ell / q \\
& =(p, s)\left(\frac{n q}{(q, s)}(q, s)^{3}(p, s)-\frac{\varepsilon \ell}{q(p, s)}\right)
\end{aligned}
$$

Here we put $a=q(p, s) /(q, s), b=(q, s)$ and $c=\ell /(q(p, s))$. Then $a b c=\ell$.

Claim 4.4. $a>1$.

Proof. Assume $a=1$. Then $(p, s)=1$ and $q=(q, s)$. Since $s=(p, s)(q, s)$, $s=(q, s)$. Thus $s=q$, so $p=r$, a contradiction.

Claim 4.5. $(a, b)=(a, c)=1$.

Proof. First, $(p, s)$ and $(q, s)$ are coprime. Also, $q /(q, s)$ and $(q, s)$ are coprime, for otherwise $(r, s)>1$. Thus $(a, b)=1$.

Next, assume $(a, c)>1$. Let $d$ be a prime factor of $(a, c)$. From Equation (4-4),

$$
n q s^{2}-p(n \ell-\varepsilon)=\varepsilon \ell / q .
$$

Dividing this by $(p, s)$ gives

$$
n q s \frac{s}{(p, s)}-\frac{p}{(p, s)}(n \ell-\varepsilon)=\varepsilon c .
$$

Since $d$ divides $a$, it divides $q$ or $s$. Similarly, $d$ divides $\ell$, since $d$ divides $c$. Thus Equation (4-6) gives

$$
\frac{p}{(p, s)} \varepsilon \equiv 0(\bmod d)
$$

However, this is impossible, because $(p, s)$ and $p /(p, s)$ are coprime. 
On the other hand, Equation (4-5) yields $p(n a b c-\varepsilon)=(p, s)\left(n a b^{3}-\varepsilon c\right)$, which, since $(p, s)$ divides $p$, means that $n a b^{3}-\varepsilon c$ is divisible by $n a b c-\varepsilon$. Furthermore

$$
\frac{n a b^{3}-\varepsilon c}{n a b c-\varepsilon}=c+\frac{n a b\left(b^{2}-c^{2}\right)}{n a b c-\varepsilon}
$$

implies that $b^{2}-c^{2}$ is divisible by $n a b c-\varepsilon$, since $n a b$ and $n a b c-\varepsilon$ are coprime.

Similarly, if $n^{2} q^{2} s^{2} \equiv-1(\bmod m)$, then $b^{2}+c^{2}$ is divisible by $n a b c-\varepsilon$. However, these are impossible by Proposition 3.1.

Proof of Corollary 1.3. Among satellite knots, only the $(2,2 p q+\varepsilon)$-cable $K$ of the $(p, q)$-torus knot admits a lens space surgery for $\varepsilon= \pm 1$. Then the slope is $4 p q+\varepsilon$, and $L\left(4 p q+\varepsilon, 4 q^{2}\right)$ arises. This surgery on $K$ is equivalent to $(4 p q+\varepsilon) / 4$-surgery on its companion torus knot. Thus the result follows from Theorem 1.2(2).

\section{Doubly primitive knot}

In this section, we study a special class of doubly primitive knots $k^{+}(a, b)$ defined by Berge [1990s]. In particular, two infinite sequences of $k^{+}(a, b)$ are proved to be hyperbolic via dual knots in lens spaces. As far as we know, whether $k^{+}(a, b)$ is hyperbolic is still an open question.

For a pair $(a, b)$ of coprime positive integers, let $k^{+}(a, b)$ denote the doubly primitive knot defined by Berge [1990s], which lies on a genus one fiber surface of the left-handed trefoil as shown in Figure 1(1). Then $\left(a^{2}+a b+b^{2}\right)$-surgery on $k^{+}(a, b)$ yields the lens space $L\left(a^{2}+a b+b^{2},(a / b)^{2}\right)$, where $a / b$ is calculated in $\mathbb{Z}_{a^{2}+a b+b^{2}}$. (We adopt the notation of [Yamada 2005], but there the orientation of lens spaces is opposite to ours). We note that $k^{+}(a, b)$ and $k^{+}(b, a)$ are equivalent by the symmetry of the fiber surface. For example, $k^{+}(1,3)$ is the $(3,4)$-torus knot whose 13 -surgery yields $L(13,9)$, and $k^{+}(2,3)$, as shown in Figure 1(2), is the $(-2,3,7)$-pretzel knot whose 19 -surgery yields $L(19,7)$.

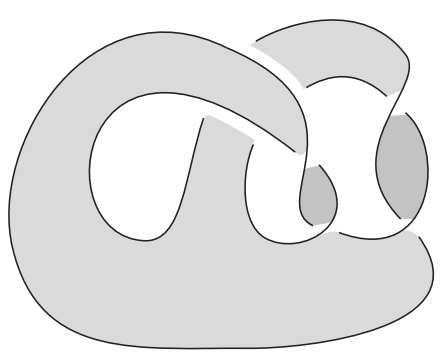

$(1)$

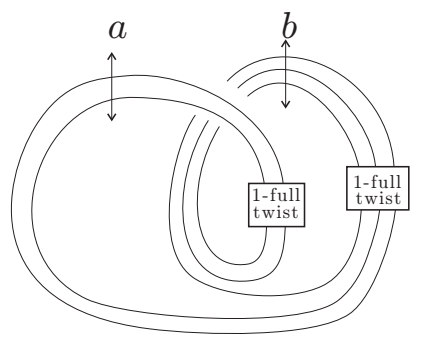

$(2)$

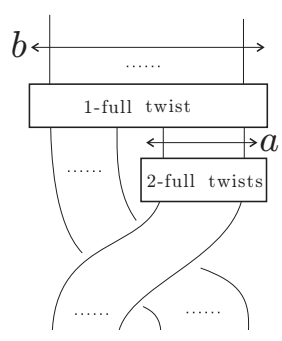

(3)

Figure 1. The knot $k^{+}(a, b)$. Check all longer captions. 
Lemma 5.1. $k^{+}(a, b)$ is a fibered knot with genus $\frac{1}{2}\left((a+b-1)^{2}-a b\right)$.

Proof. It is easy to see that $k^{+}(a, b)$ has a form of the closure of a positive braid as shown in Figure 1(3). By [Stallings 1978], Seifert's algorithm gives a fiber surface. The braid has $b$ strings and $a^{2}+a b+b^{2}-2 a-b$ crossings, so the fiber has the given genus. (See also [Yamada 2005, Corollary 3] or [Hill and Murasugi 2000].)

In general, let $K$ be a knot in $S^{3}$ whose $p$-surgery yields $L(p, q)$ with $p>$ $q>0$. Then the core $K^{*}$ of the attached solid torus of $K(p)$ is called the dual knot of $K$ (with respect to $p$-surgery). Berge [1990s] shows that if $K$ is a doubly primitive knot whose surface slope is $p$, then $K^{*}$ is a $(1,1)$-knot in $L(p, q)$ and has a canonical form parametrized by a single integer $k$ with $0<k<p$ (see [Saito 2007; 2008b]). Following [Saito 2007], we denote it by $K(L(p, q) ; k)$. It is known that $K(L(p, q) ; k)$ is isotopic to $K(L(p, q) ; p-k)$.

For $n=1,2, \ldots, p-1$, let $\phi_{n}$ be an integer such that $\phi_{n} \equiv n q(\bmod p)$ and $0<\phi_{n}<p$. We call this finite sequence $\left\{\phi_{n}\right\}$ the basic sequence for $(p, q)$. Because of $\operatorname{gcd}(p, q)=1$, the $\phi_{n}$ are mutually distinct. In particular, $k$ appears in the basic sequence. Let $h$ be the position of $k$, that is, $\phi_{h}=k$. Here, set

$$
\begin{aligned}
s & =\sharp\{i \mid i<k \text { and } i \text { appears before } k \text { in the basic sequence }\}, \\
\ell & =\sharp\{i \mid i>k \text { and } i \text { appears before } k \text { in the basic sequence }\}, \\
s^{\prime} & =\sharp\{i \mid i<k \text { and } i \text { appears after } k \text { in the basic sequence }\}, \\
\ell^{\prime} & =\sharp\{i \mid i>k \text { and } i \text { appears after } k \text { in the basic sequence }\} .
\end{aligned}
$$

Let $\Phi=\min \left\{s, s^{\prime}, \ell, \ell^{\prime}\right\}$. This is determined for the triplet $(p, q, k)$ and so also for the dual knot $K(L(p, q) ; k)$. However, the main result of [Saito 2008a] says that $\Phi$ depends only on the original knot $K$ and a lens space surgery slope $p$, and that $K$ is hyperbolic if and only if $\Phi \geq 2$ or equivalently each of $s, s^{\prime}, \ell, \ell^{\prime}$ is at least two.

For $k^{+}(a, b)$, let $p=a^{2}+a b+b^{2}$. Then $p$-surgery yields a lens space $L(p, q)$ where $q \equiv(b /(a+b))^{2}$; note that $(a / b)^{2} \equiv(b /(a+b))^{2}(\bmod p)$. By [Saito 2007], the dual knot is represented as $K(L(p, q) ; k)$ with $k \equiv-b /(a+b)(\bmod p)$. (By definition, the parameter $k$ is chosen so that $0<k<p$.)

Lemma 5.2. Let $p, q$ and $k$ be defined as above.

(1) $k+q+1 \equiv 0(\bmod p)$.

(2) $k \equiv q^{2}(\bmod p)$.

(3) $k q \equiv 1(\bmod p)$. 
Proof. (1) We compute

$$
\begin{aligned}
k+q+1 & \equiv-\frac{b}{a+b}+\frac{b^{2}}{(a+b)^{2}}+1=\frac{-b(a+b)+b^{2}+(a+b)^{2}}{(a+b)^{2}} \\
& =\frac{(a+b)^{2}-a b}{(a+b)^{2}}=\frac{p}{(a+b)^{2}} \equiv 0(\bmod p) .
\end{aligned}
$$

(2) $\mathrm{By}(1), q^{2}-k \equiv(-k-1)^{2}-k=k^{2}+k+1 \equiv q+k+1 \equiv 0(\bmod p)$.

(3) Similarly, $k q \equiv k(-k-1)=-k^{2}-k \equiv-q-k \equiv 1(\bmod p)$ by (1).

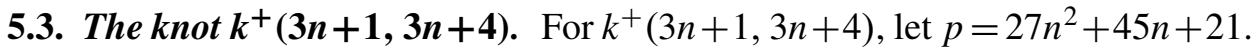
Then $p$-surgery yields a lens space $L(p, q)$ with $q=(3 n+2)^{2}$, and the dual knot is $K(L(p, q) ; k)$ with $k \equiv-(3 n+2)^{2}-1(\bmod p)$.

Lemma 5.4. The knot $k^{+}(3 n+1,3 n+4)$ is hyperbolic for $n \geq 1$.

Proof. Let $a=3 n+1, b=3 n+4$ and $k_{0}=p-q-1$. Then direct calculations show that $3 q<p<4 q, k_{0} \equiv k(\bmod p)$ and $2 q-1<k_{0}<3 q-a$. Thus we can use the triplet $\left(p, q, k_{0}\right)$ to calculate the invariant $\Phi$.

Let $\left\{\phi_{i}\right\}$ be the basic sequence. (Recall that any term $\phi_{i}$ of the basic sequence is chosen so that $0<\phi_{i}<p$.) Since $q^{2} \equiv k \equiv k_{0}(\bmod p)$ by Lemma 5.2, $\phi_{q}=k_{0}$.

First, we study the four consecutive terms $\phi_{a+b}, \phi_{a+b+1}, \phi_{a+b+2}, \phi_{a+b+3}$, which appear before $k_{0}$. Since $(a+b) q \equiv p-a(\bmod p)$, we have $\phi_{a+b}=p-a$. Then

$$
q-a<2 q-a<k_{0}<3 q-a<p-a,
$$

so

$$
\phi_{a+b+1}=q-a, \quad \phi_{a+b+2}=2 q-a, \quad \phi_{a+b+3}=3 q-a .
$$

Hence

$$
\phi_{a+b}>k_{0}, \quad \phi_{a+b+1}<k_{0}, \quad \phi_{a+b+2}<k_{0}, \quad \phi_{a+b+3}>k_{0} .
$$

Similarly, we study the four consecutive terms right after $k_{0}$. (Since $q+4<p-1$, there are more than four terms after $k_{0}$.) Since $k_{0}+q=p-1$, we have $\phi_{q+1}=p-1$. Then

$$
\phi_{q+2}=q-1, \quad \phi_{q+3}=2 q-1, \quad \phi_{q+4}=3 q-1 .
$$

Hence

$$
\phi_{q+1}>k_{0}, \quad \phi_{q+2}<k_{0}, \quad \phi_{q+3}<k_{0}, \quad \phi_{q+4}>k_{0} .
$$

Thus $\Phi \geq 2$, showing that the dual knot (and the original knot) is hyperbolic.

5.5. The $\boldsymbol{k n o t} \boldsymbol{k}^{+}\left(\boldsymbol{F}_{\boldsymbol{n}+\mathbf{2}}, \boldsymbol{F}_{\boldsymbol{n}}\right)$. For Fibonacci numbers, see Section 2 . Let $p=$ $F_{n}^{2}+F_{n} F_{n+2}+F_{n+2}^{2}$. Then $p$-surgery on $k^{+}\left(F_{n+2}, F_{n}\right)$ yields a lens space $L(p, q)$ with $q \equiv\left(F_{n} /\left(F_{n+2}+F_{n}\right)\right)^{2}(\bmod p)$. Denote the dual knot by $K(L(p, q) ; k)$.

Lemma 5.6. We have $p=4 F_{n} F_{n+2}+(-1)^{n}, q \equiv(-1)^{n+1} 4 F_{n}^{2}(\bmod p)$, and $k \equiv(-1)^{n} 4 F_{n}\left(F_{n}+F_{n+2}\right)(\bmod p)$. 
Proof. By Cassini's identity $F_{n} F_{n+2}-F_{n+1}^{2}=(-1)^{n+1}$,

$$
\begin{aligned}
4 F_{n} F_{n+2}+(-1)^{n} & =4 F_{n+1}^{2}+3(-1)^{n+1} \\
& =3\left(F_{n+1}^{2}+(-1)^{n+1}\right)+F_{n+1}^{2} \\
& =3 F_{n} F_{n+2}+F_{n+1}^{2} \\
& =F_{n} F_{n+2}+2 F_{n}\left(F_{n}+F_{n+1}\right)+F_{n+1}^{2} \\
& =F_{n} F_{n+2}+2 F_{n}^{2}+2 F_{n} F_{n+1}+F_{n+1}^{2} \\
& =F_{n} F_{n+2}+F_{n}^{2}+\left(F_{n}+F_{n+1}\right)^{2}=F_{n} F_{n+2}+F_{n}^{2}+F_{n+2}^{2}=p .
\end{aligned}
$$

Thus $4 F_{n} F_{n+2}+(-1)^{n} \equiv 0(\bmod p)$. To show $q \equiv(-1)^{n+1} 4 F_{n}^{2}(\bmod p)$, it suffices to show $(-1)^{n+1} 4\left(F_{n}+F_{n+2}\right)^{2} \equiv 1(\bmod p)$. This follows from the equation $\left(F_{n}+F_{n+2}\right)^{2} \equiv F_{n} F_{n+2}(\bmod p)$.

Finally,

$$
(-1)^{n} 4 F_{n}\left(F_{n}+F_{n+2}\right)=(-1)^{n} 4\left(F_{n}^{2}+F_{n} F_{n+2}\right) \equiv(-1)^{n+1} 4 F_{n+2}^{2}(\bmod p) .
$$

Then $(-1)^{n+1} 4 F_{n+2}^{2} q \equiv\left(4 F_{n+2} F_{n}\right)^{2} \equiv 1(\bmod p)$. This shows by Lemma 5.2(3) that $(-1)^{n+1} 4 F_{n}\left(F_{n}+F_{n+2}\right) \equiv 1 / q \equiv k(\bmod p)$.

Lemma 5.7. For $n \geq 3$, the knot $k^{+}\left(F_{n+2}, F_{n}\right)$ is hyperbolic.

Proof. As mentioned above, $p$-surgery on $k^{+}\left(F_{n+2}, F_{n}\right)$ yields $L(p, q)$. Consider the dual knot $K(L(p, q) ; k)$ in $L(p, q)$.

First, we assume that $n$ is odd. Then $p=4 F_{n} F_{n+2}-1, q \equiv 4 F_{n}^{2}(\bmod p)$, and $k \equiv-4 F_{n}\left(F_{n}+F_{n+2}\right)(\bmod p)$ by Lemma 5.6.

To simplify calculation of the invariant $\Phi$, put $q_{0}=p-4 F_{n}^{2}$ and $k_{0}=p-q_{0}+1$. Then $0<q_{0}<p$ and $0<k_{0}<p$, and $q_{0} \equiv-q(\bmod p)$ and $k_{0} \equiv-k(\bmod p)$.

Claim 5.8. $3 q_{0} / 2<p<2 q_{0}$ and $2 q_{0}-p<k_{0}<q_{0}$.

Proof. $2 q_{0}-p=p-8 F_{n}^{2}=4 F_{n} F_{n+2}-1-8 F_{n}^{2}=4 F_{n}\left(F_{n}+F_{n+1}\right)-8 F_{n}^{2}-1=$ $4 F_{n}\left(F_{n+1}-F_{n}\right)-1 \geq 7$. Since $3 F_{n}>F_{n+2}$, we have $2 p-3 q_{0}=12 F_{n}^{2}-p=$ $4 F_{n}\left(3 F_{n}-F_{n+2}\right)+1 \geq 9$.

Next, $k_{0}-2 q_{0}+p=2 p-3 q_{0}+1 \geq 10$. Finally, $q_{0}-k_{0}=2 q_{0}-p-1 \geq 6$.

For $\left(p, q_{0}\right)$, let $\left\{\phi_{i}\right\}$ be the basic sequence; let $h=p-q_{0}$. As $h q_{0} \equiv k_{0}(\bmod p)$, the number $k_{0}$ appears as the $h$-th term in the sequence. Note that $h>4$, because $2 p-3 q_{0} \geq 9$.

To evaluate $\Phi$, we investigate some specific terms in the basic sequence. We have $\phi_{1}=q_{0}>k_{0}$ and $\phi_{2}=2 q_{0}-p<k_{0}$. Also, $\phi_{h-1}=k_{0}-q_{0}+p>k_{0}$ and $\phi_{h-2}=k_{0}-2 q_{0}+p<k_{0}$. Since $h>4$, these four terms $\phi_{1}, \phi_{2}, \phi_{h-2}, \phi_{h-1}$ are distinct. Next, $\phi_{p-1}=p-q_{0}<k_{0}$ and $\phi_{p-2}=2 p-2 q_{0}>k_{0}$. Since $2 h<p$, $h<p-h+1$. Thus $\phi_{p-h+1}$ and $\phi_{p-h+2}$, which are distinct from $\phi_{p-1}$ and $\phi_{p-2}$, 


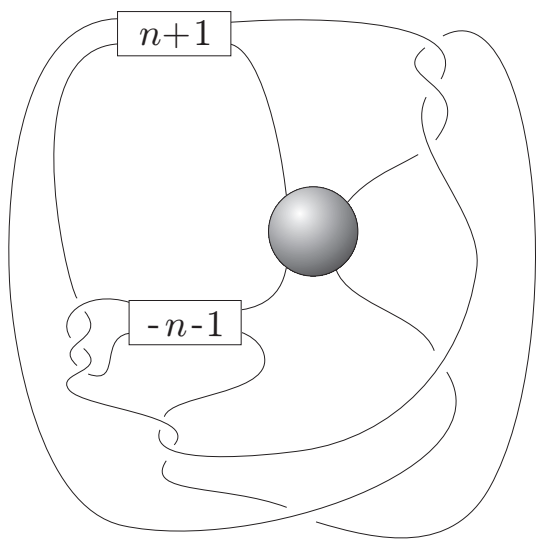

Figure 2. The tangle $B_{n}$.

appear after $k_{0}$ in the basic sequence. Since $(p-h+1) q_{0} \equiv q_{0}-k_{0}(\bmod p)$, we have $\phi_{p-h+1}=q_{0}-k_{0}$. Then $k_{0}-\left(q_{0}-k_{0}\right)=2 k_{0}-q_{0}=2 p-3 q_{0}+2>0$ implies $\phi_{p-h+1}<k_{0}$. Finally, $\phi_{p-h+2}=2 q_{0}-k_{0}>k_{0}$. Again, the fact $h>4$ means that the four terms $\phi_{p-h+1}, \phi_{p-h+2}, \phi_{p-2}, \phi_{p-1}$ are distinct. Hence $\Phi \geq 2$.

Second, assume that $n$ is even. Then $p=4 F_{n} F_{n+2}+1, q \equiv-4 F_{n}^{2}(\bmod p)$, and $k \equiv 4 F_{n}\left(F_{n}+F_{n+2}\right)(\bmod p)$ by Lemma 5.6. In this case, put $q_{0}=p-4 F_{n}^{2}+1$ and $k_{0}=p-q_{0}+1$. Then $0<q_{0}<p$ and $0<k_{0}<p$. It is easy to check that Claim 5.8 holds without any change.

By Lemma 5.2, $q_{0} q \equiv(q+1) q \equiv k+q \equiv-1(\bmod p)$ and $k_{0} \equiv-q(\bmod p)$. Under a (orientation-reversing) homeomorphism from $L(p, q)$ to $L\left(p, q_{0}\right)$, the dual knot $K(L(p, q) ; k)$ is mapped to $K\left(L\left(p, q_{0}\right) ; k_{0}\right)$; see [Saito 2008a]. Thus we can use $\left(p, q_{0}, k_{0}\right)$ instead of $(p, q, k)$ to evaluate $\Phi$.

By Lemma $5.2(2), q^{2} \equiv k(\bmod p)$. Thus $16 F_{n}^{4} \equiv 4 F_{n}\left(F_{n}+F_{n+2}\right)$. Hence $q_{0}^{2}+k_{0} \equiv\left(1-4 F_{n}^{2}\right)^{2}+4 F_{n}^{2} \equiv 16 F_{n}^{4}-4 F_{n}^{2}+1 \equiv 4 F_{n}\left(F_{n}+F_{n+2}\right)-4 F_{n}^{2}+1 \equiv$ $4 F_{n}^{2} F_{n+2}^{2}+1 \equiv 0(\bmod p)$. This means that $\left(p-q_{0}\right) q_{0} \equiv k_{0}(\bmod p)$. Let $h=p-q_{0}$. Then, $k_{0}$ appears in the basic sequence for $\left(p, q_{0}\right)$ as the $h$-term. Since $h>4$, the argument in the case where $n$ is odd works verbatim, so we have $\Phi \geq 2$.

\section{Hyperbolic knots}

We say a Seifert-fibered manifold is of type $X\left(p_{1}, p_{2}, \ldots, p_{n}\right)$ if it admits a Seifert fibration over the surface $X$ with $n$ exceptional fibers of indices $p_{1}, p_{2}, \ldots, p_{n}$. In this paper, $X$ will be either the 2-sphere $S^{2}$ or the disk $D^{2}$.

For $n \geq 1$, let $B_{n}$ be the tangle illustrated in Figure 2, in which a rectangle denotes horizontal half-twists. If the number is positive, the twist is right-handed; otherwise, it is left-handed. 


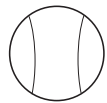

$1 / 0$

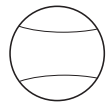

0

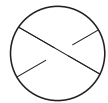

$-1$

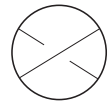

1

Figure 3. Some rational tangles.

Given $\alpha \in \mathbb{Q} \cup\{1 / 0\}$, we denote by $B_{n}(\alpha)$ the knot or link in $S^{3}$ obtained by inserting the rational tangle of slope $\alpha$ into the central puncture of $B_{n}$. Also, $\widetilde{B}_{n}$ is the double branched cover of $S^{3}$ branched over $B_{n}(\alpha)$. In fact, we need only four rational tangles as shown in Figure 3.

Lemma 6.1. (1) $\widetilde{B}_{n}(1 / 0)=S^{3}$.

(2) $\widetilde{B}_{n}(0)=L\left(27 n^{2}+45 n+21,-9 n^{2}-12 n-5\right)$.

(3) $\widetilde{B}_{n}(1)$ is a Seifert fibered manifold of type $S^{2}(2, n+2,15 n+11)$.

(4) $\widetilde{B}_{n}(-1)$ is a non-Seifert toroidal manifold $D^{2}(2, n) \cup D^{2}(2,3 n+1)$, which contains a unique incompressible torus if $n \geq 2$, or a Seifert fibered manifold of type $S^{2}(2,3,4)$ if $n=1$.

Proof. It is straightforward to check that $B_{n}(1 / 0)$ is the unknot and that $B_{n}(0)$ is the 2-bridge knot corresponding to $-\left(9 n^{2}+12 n+5\right) /\left(27 n^{2}+45 n+21\right)$.

Figure 4 shows that $B_{n}(1)$ is a Montesinos link or knot of length three. Thus $\widetilde{B}_{n}(1)$ is a Seifert fibered manifold of type $S^{2}(2, n+2,15 n+11)$.

Figure 5 shows that $B_{n}(-1)$ is decomposed along a tangle sphere $P$ into two tangles. If $n>1$, then each side of $P$ is a Montesinos tangle. Thus $\widetilde{B}_{n}(1)$ is decomposed along a torus into two Seifert fibered manifolds over the disk with two exceptional fibers. Since Seifert fibers on both sides intersect once on the torus, $\widetilde{B}_{n}(-1)$ is not Seifert. It is well known that such a 3 -manifold contains a
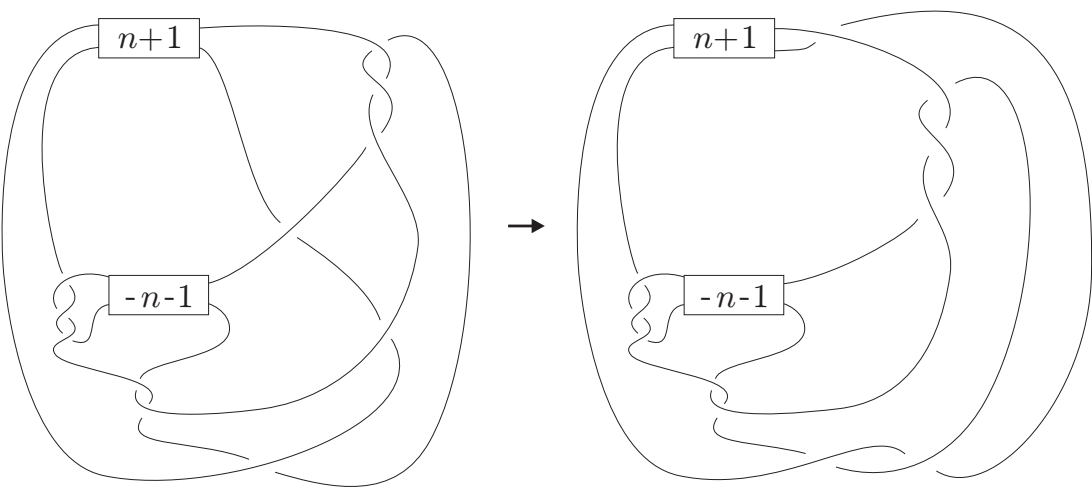

Figure 4. $B_{n}(1)$, a Montesinos link. 


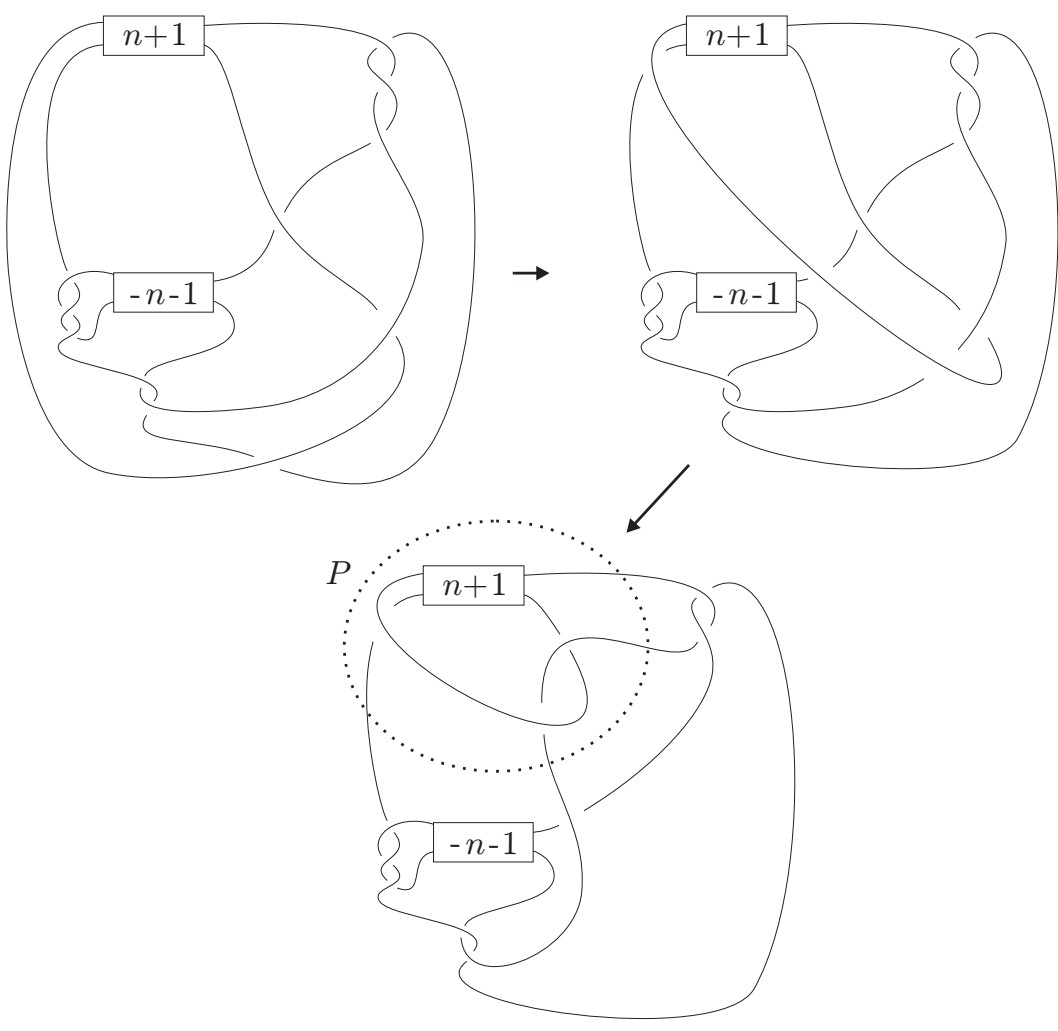

Figure 5. $B_{n}(-1)$.

unique incompressible torus. When $n=1, B_{n}(-1)$ is a Montesinos link of length three. Hence $\widetilde{B}_{n}(-1)$ is a Seifert fibered manifold over the 2 -sphere with three exceptional fibers.

By Lemma 6.1(1), the lift of $B_{n}$ in $\widetilde{B}_{n}(1 / 0)$ gives the knot exterior of some knot $K_{n}$ in $S^{3}$, which is uniquely determined by Gordon and Luecke's theorem [1989]. Furthermore, $K_{n}$ admits integral Dehn surgeries yielding a lens space, a Seifert fibered manifold, and a toroidal manifold (unless $n=1$ ) by Lemma 6.1.

The following criterion of hyperbolicity is used also in Section 7.

Lemma 6.2. If a knot $K$ in $S^{3}$ admits an integral lens space surgery $m$, and neither $K(m-1)$ nor $K(m+1)$ has a lens space summand, then $K$ is hyperbolic.

Proof. Assume the contrary. Then $K$ is either a torus knot or a satellite knot. For the (nontrivial) $(p, q)$-torus knot, the only integral lens space surgery slopes are $p q-1$ and $p q+1$, and $p q$-surgery yields the connected sum of two lens spaces by [Moser 1971]. Thus $K$ is not a torus knot. 
Assume $K$ is a satellite knot. Since $K$ has a lens space surgery, we know by [Bleiler and Litherland 1989; Wang 1989; Wu 1990] that $K$ is the $(2,2 p q+\varepsilon)$ cable of the $(p, q)$-torus knot where $\varepsilon \in\{1,-1\}$. Then the lens space surgery is $4 p q+\varepsilon$. However, the adjacent slope $4 p q+2 \varepsilon$ is equal to the cabling slope, and so $K(4 p q+2 \varepsilon)$ has a lens space summand, a contradiction. Thus $K$ is hyperbolic.

Lemma 6.3. $K_{n}$ is hyperbolic.

Proof. This immediately follows from Lemmas 6.1 and 6.2.

Lemma 6.4. The knot $K_{n}$ defined above satisfies the following.

(1) The genus of $K_{n}$ is $\left(27 n^{2}+33 n+10\right) / 2$.

(2) Let $m=27 n^{2}+45 n+21$. Then $m$-surgery on $K$ yields the lens space $L\left(m,-9 n^{2}-12 n-5\right)$.

Proof. Insert the 1/0-tangle to $B_{n}$, and put a band $b$ as shown in Figure 6 to keep track of framing. Isotope the unknot $B_{n}(1 / 0)$ to a standard diagram as shown in Figure 8 (in which the cases $n=5$ and $n=4$ are drawn), and take the double branched cover along it. Then (the core of) the lift of $b$ gives $K_{n}$, and its framing corresponds to the 0 -tangle filling downstairs. (In Figures 6, 7 and 8, we draw $b$ in a line for simplicity during the deformation.) From Figure 8, we see that $K_{n}$ is the closure of a braid with $3 n+2$ strings. Moreover, there are $27 n^{2}+41 n+10$ positive crossings and $5 n-1$ negative crossings. After canceling the negative crossings by positive crossings, $K_{n}$ becomes the closure of a positive braid with $3 n+2$ strings and $27 n^{2}+36 n+11$ crossings. By [Stallings 1978], $K_{n}$ is fibered and Seifert's algorithm gives a fiber surface, whose genus is equal to the genus $g\left(K_{n}\right)$ of $K_{n}$. Now (1) follows because $1-2 g\left(K_{n}\right)=(3 n+2)-\left(27 n^{2}+36 n+11\right)$.

The framing of the lift of $b$ can be calculated to equal $m$. This proves (2).
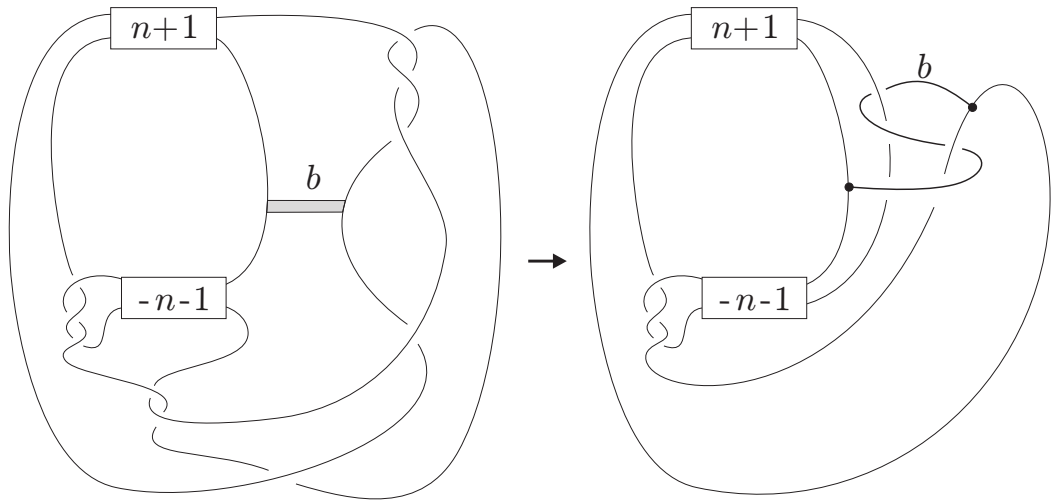

Figure 6. $B_{n}(1 / 0)$ and the band $b$. 

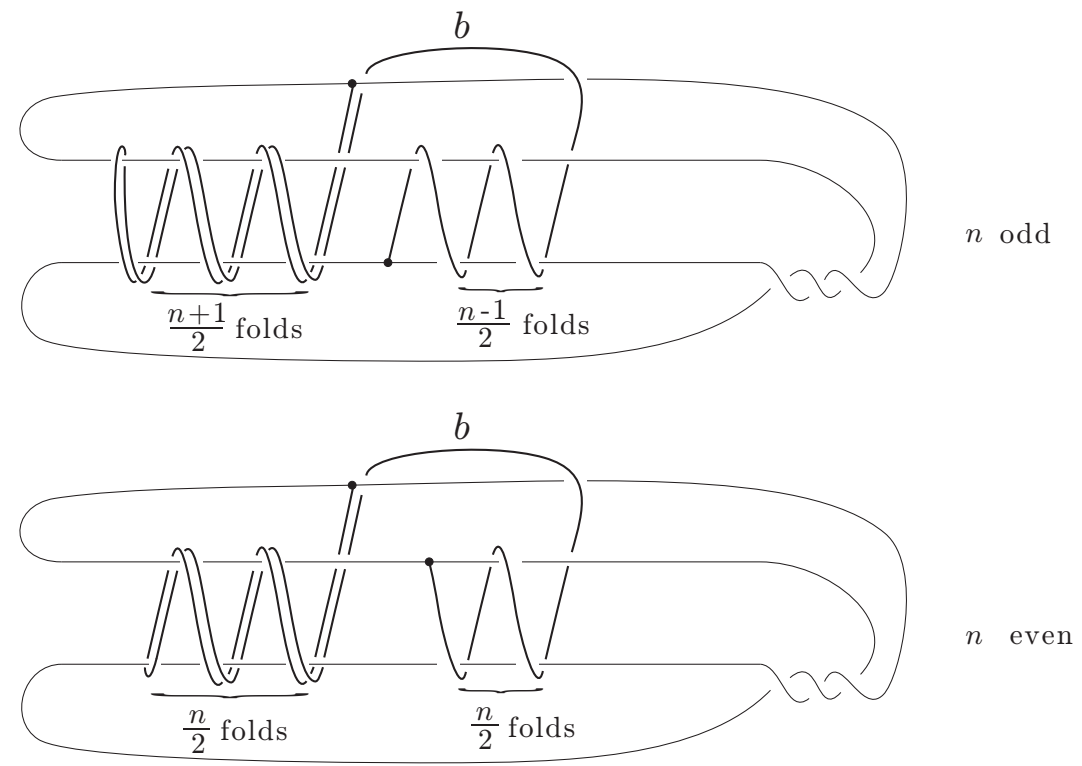

Figure 7. $B_{n}(1 / 0)$ with $b$.

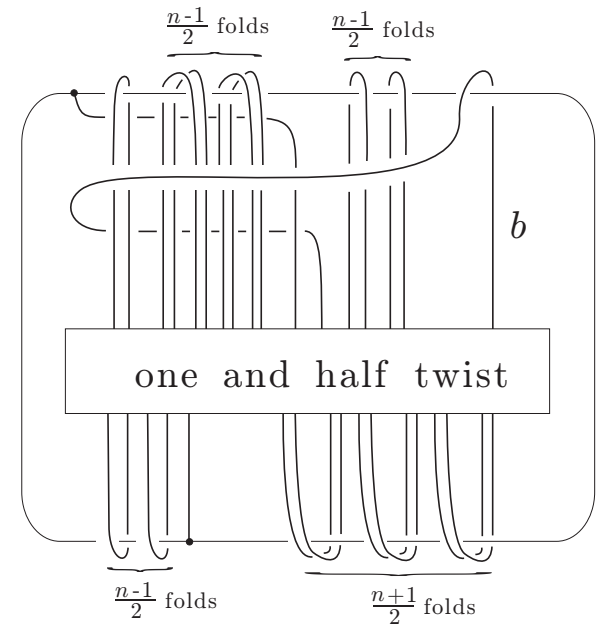

$n$ odd

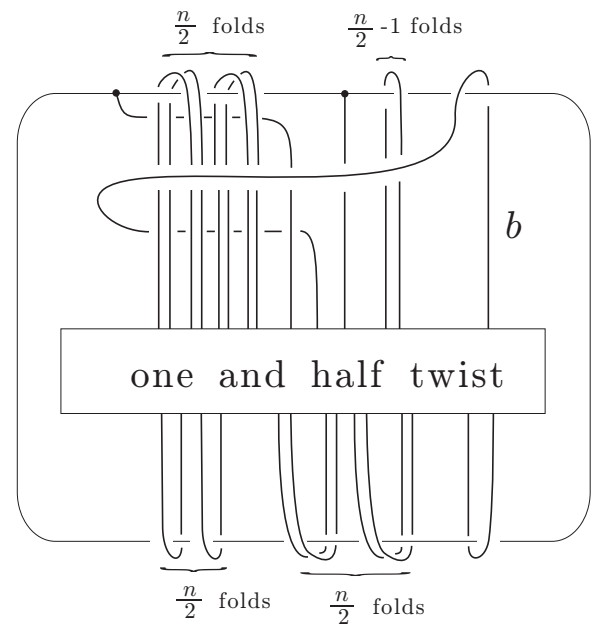

$n$ even

Figure 8. The standard diagram of $B_{n}(1 / 0)$ with $b$.

Recall that $k^{+}(3 n+1,3 n+4)$ is hyperbolic for $n \geq 1$ by Lemma 5.4.

Proposition 6.5. For $n \geq 1$, let $K$ be the hyperbolic knot $K_{n}$ defined above, and let $K^{\prime}$ be $k^{+}(3 n+1,3 n+4)$. Let $m=27 n^{2}+45 n+21$. Then $K$ and $K^{\prime}$ are not equivalent, and $m$-surgery on $K$ and $K^{\prime}$ yield homeomorphic lens spaces. 
Proof. By Lemma 6.4(1), $K$ has genus $\left(27 n^{2}+33 n+10\right) / 2$, while $K^{\prime}$ has genus $\left(27 n^{2}+33 n+12\right) / 2$ by Lemma 5.1 . Thus they are not equivalent.

Also, by Lemma 6.4(2), $m$-surgery on $K$ yields $L\left(m,-9 n^{2}-12 n-5\right)=$ $L\left(m, 18 n^{2}+33 n+16\right)$. As we stated in Section $5, m$-surgery on $K^{\prime}$ yields $L\left(m,((3 n+1) /(3 n+4))^{2}\right)$. Those lens spaces are homeomorphic since

$$
\left(\frac{3 n+1}{3 n+4}\right)^{2}\left(18 n^{2}+33 n+16\right) \equiv 1(\bmod m) .
$$

\section{Different classes}

In this last section, we give pairs of knots, each of which yields homeomorphic lens spaces by the same integral surgery, and consist of knots belonging to different classes of hyperbolic, satellite, torus knots.

7.1. Torus knot and satellite knot. Let $C(a, b)$ be the $(2,2 a b+1)$-cable of the torus knot of type $(a, b)$.

Proposition 7.2. For $n \geq 1$, let $K$ be the torus knot of type $(2 n+1,4 n+4)$, and let $K^{\prime}=C(n+1,2 n+1)$. Let $m=8 n^{2}+12 n+5$. Then $m$-surgery on $K$ and $K^{\prime}$ yields homeomorphic lens spaces.

Proof. By [Moser 1971], $m$-surgery on $K$ yields the lens space $L\left(m,(2 n+1)^{2}\right)$. Also, $m$-surgery on $K^{\prime}$ yields $L\left(m, 4(n+1)^{2}\right)$ by [Fintushel and Stern 1980]. Since $(2 n+1)^{2}+4(n+1)^{2}=m$, these lens spaces are homeomorphic.

\subsection{Satellite knot and hyperbolic knot.}

Lemma 7.4. For $n \geq 0$,

$$
4 F_{n}^{4}+(-1)^{n} F_{n+2}^{2}=\left(4 F_{n} F_{n+2}+(-1)^{n}\right)\left(F_{n+2}^{2}-4 F_{n} F_{n+1}\right) .
$$

Proof. First,

$$
\begin{aligned}
4 F_{n}^{4}+(-1)^{n} F_{n+2}^{2}-\left(4 F_{n} F_{n+2}\right. & \left.+(-1)^{n}\right)\left(F_{n+2}^{2}-4 F_{n} F_{n+1}\right) \\
= & 4 F_{n}\left(F_{n}^{3}-F_{n+2}^{3}+4 F_{n} F_{n+1} F_{n+2}-(-1)^{n+1} F_{n+1}\right) .
\end{aligned}
$$

From Cassini’s identity,

$$
\begin{aligned}
& F_{n}^{3}-F_{n+2}^{3}+4 F_{n} F_{n+1} F_{n+2}-(-1)^{n+1} F_{n+1} \\
= & F_{n}^{3}-F_{n+2}^{3}+3 F_{n} F_{n+1} F_{n+2}+F_{n+1}^{3} \\
= & F_{n}^{3}-\left(F_{n}+F_{n+1}\right)^{3}+3 F_{n} F_{n+1} F_{n+2}+F_{n+1}^{3} \\
= & -3 F_{n} F_{n+1}\left(F_{n}+F_{n+1}-F_{n+2}\right)=0 .
\end{aligned}
$$




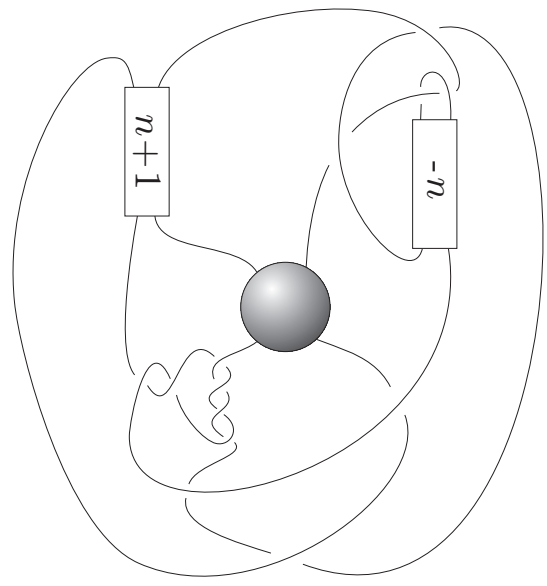

Figure 9. The tangle $B_{n}$.

Since $\operatorname{gcd}\left(F_{n}, F_{n+2}\right)=\operatorname{gcd}\left(F_{n}, F_{n+1}\right)=1$, the Fibonacci numbers $F_{n}$ and $F_{n+2}$ are coprime. By Lemma 5.7, $k^{+}\left(F_{n+2}, F_{n}\right)$ is hyperbolic for $n \geq 3$.

Proposition 7.5. For $n \geq 3$, let $K$ be the satellite knot $C\left(F_{n}, F_{n+2}\right)$, and let $K^{\prime}$ be the hyperbolic knot $k^{+}\left(F_{n+2}, F_{n}\right)$. Let $m=4 F_{n} F_{n+2}+(-1)^{n}$. Then $m$-surgery on $K$ and $K^{\prime}$ yields homeomorphic lens spaces.

Proof. By [Fintushel and Stern 1980], $m$-surgery on $K$ yields the lens space $L\left(m, 4 F_{n}^{2}\right)$. From Lemma 5.6, $m$-surgery on $K^{\prime}$ yields $L\left(m,\left(F_{n} / F_{n+2}\right)^{2}\right)$. Then

$$
4 F_{n}^{2}\left(\frac{F_{n}}{F_{n+2}}\right)^{2} \equiv(-1)^{n+1}(\bmod m)
$$

by Lemma 7.4, Thus the two lens spaces are homeomorphic.

7.6. Torus knot and hyperbolic knot. For $n \geq 1$, let $B_{n}$ be the tangle as shown in Figure 9, where a vertical box denotes right-handed vertical half-twists.

Given $\alpha \in \mathbb{Q} \cup\{1 / 0\}$, we denote by $B_{n}(\alpha)$ the knot or link in $S^{3}$ obtained by inserting the rational tangle of slope $\alpha$ into the central puncture of $B_{n}$. Also, $\widetilde{B}_{n}$ is the double branched cover of $S^{3}$ branched over $B_{n}(\alpha)$.

Lemma 7.7. (1) $\widetilde{B}_{n}(1 / 0)=S^{3}$.

(2) $\widetilde{B}_{n}(0)=L\left(18 n^{2}+33 n+15,18 n+19\right)$.

(3) $\widetilde{B}_{n}(-1)$ is a non-Seifert toroidal manifold $D^{2}(2, n+2) \cup D^{2}(4,2 n+1)$.

(4) $\widetilde{B}_{n}(1)$ is a non-Seifert toroidal manifold $D^{2}(2, n) \cup D^{2}(5,2 n+3)$ if $n \geq 2$, and a Seifert fibered manifold of type $S^{2}(3,5,5)$ if $n=1$. 


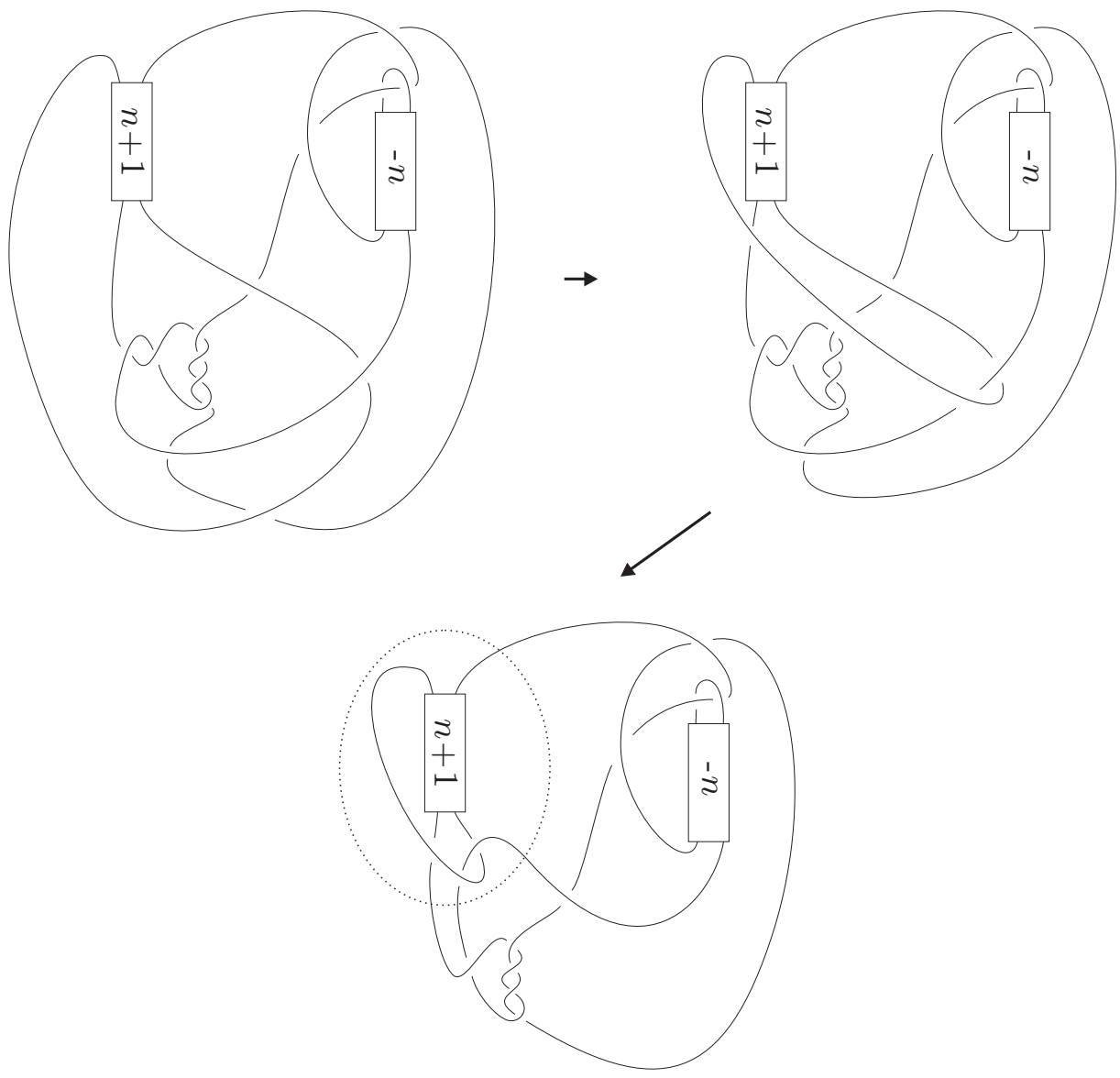

Figure 10. $B_{n}(-1)$.

Proof. It is straightforward to see that $B(1 / 0)$ is the unknot and $B(0)$ is the 2-bridge link corresponding to $\left(18 n^{2}+33 n+15\right) /(18 n+19)$. For $B(-1)$ and $B(1)$, see Figures 10 and 11, respectively.

By Lemma 7.7(1), the lift of $B_{n}$ in $\widetilde{B}_{n}(1 / 0)$ gives the knot exterior of some knot $K_{n}$ in $S^{3}$, which is uniquely determined by Gordon and Luecke's theorem [1989].

Lemma 7.8. $K_{n}$ is hyperbolic.

Proof. This immediately follows from Lemmas 6.2 and 7.7.

Lemma 7.9. Let $m=18 n^{2}+33 n+15$. Then $m$-surgery on $K_{n}$ yields the lens space $L(m,-18 n-19)$.

Proof. The argument is similar to the proof of Lemma 6.4. We omit it. 

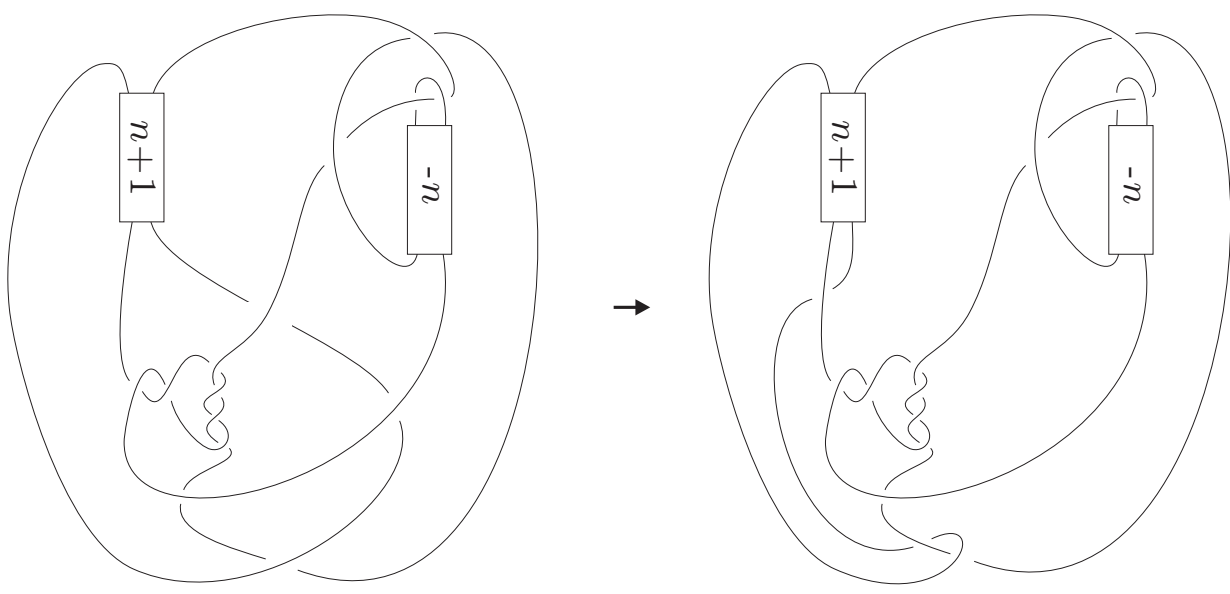

Figure 11. $B_{n}(1)$.

Proposition 7.10. For $n \geq 1$, let $K$ be the torus knot of type $(3 n+2,6 n+7)$, and let $K^{\prime}$ be the knot $K_{n}$ defined above. Let $m=18 n^{2}+33 n+15$. Then $m$-surgery on $K$ and $K^{\prime}$ yields homeomorphic lens spaces.

Proof. By [Moser 1971], $m$-surgery on $K$ yields $L\left(m, 9 n^{2}+12 n+4\right)$. Then by Lemma $7.9, m$-surgery on $K^{\prime}$ yields $L(m, 18 n+19)$. Since

$$
\left(9 n^{2}+12 n+4\right)(18 n+19) \equiv 1(\bmod m),
$$

two lens spaces are homeomorphic.

Theorem 1.1 now follows from Propositions 2.2, 6.5, 7.2, 7.5 and 7.10.

\section{Acknowledgment}

We would like to thank Kazuhiro Kawasaki for performing computer experiments.

\section{References}

[Berge 1990s] J. Berge, "Some knots with surgeries yielding lens spaces", unpublished manuscript, 1990s.

[Bleiler and Litherland 1989] S. A. Bleiler and R. A. Litherland, "Lens spaces and Dehn surgery", Proc. Amer. Math. Soc. 107:4 (1989), 1127-1131. MR 90e:57031 Zbl 0686.57007

[Brakes 1980] W. R. Brakes, "Manifolds with multiple knot-surgery descriptions", Math. Proc. Cambridge Philos. Soc. 87:3 (1980), 443-448. MR 81b:57002 Zbl 0443.57010

[Culler et al. 1987] M. Culler, C. M. Gordon, J. Luecke, and P. B. Shalen, "Dehn surgery on knots", Ann. of Math. (2) 125:2 (1987), 237-300. MR 88a:57026 Zbl 0633.57006

[Fintushel and Stern 1980] R. Fintushel and R. J. Stern, "Constructing lens spaces by surgery on knots”, Math. Z. 175:1 (1980), 33-51. MR 82i:57009a Zbl 0425.57001 
[Flath 1989] D. E. Flath, Introduction to number theory, Wiley, New York, 1989. MR 90c:11001 Zbl 0651.10001

[Gordon and Luecke 1989] C. M. Gordon and J. Luecke, "Knots are determined by their complements", J. Amer. Math. Soc. 2:2 (1989), 371-415. MR 90a:57006a Zbl 0678.57005

[Graham et al. 1994] R. L. Graham, D. E. Knuth, and O. Patashnik, Concrete mathematics: A foundation for computer science, 2nd ed., Addison-Wesley, Reading, MA, 1994. MR 97d:68003 Zbl 0836.00001

[Hill and Murasugi 2000] P. Hill and K. Murasugi, "On double-torus knots, II", J. Knot Theory Ramifications 9:5 (2000), 617-667. MR 2001f:57006 Zbl 0991.57011

[Kawauchi 1996] A. Kawauchi, "Mutative hyperbolic homology 3-spheres with the same Floer homology", Geom. Dedicata 61:2 (1996), 205-217. MR 97g:57020 Zbl 0857.57012

[Kronheimer et al. 2007] P. Kronheimer, T. Mrowka, P. Ozsváth, and Z. Szabó, "Monopoles and lens space surgeries”, Ann. of Math. (2) 165:2 (2007), 457-546. MR 2008b:57037 Zbl 05180741

[Lickorish 1976] W. B. R. Lickorish, "Surgery on knots", Proc. Amer. Math. Soc. 60 (1976), 296298. MR 58 \#7632 Zbl 0345.57002

[Livingston 1982] C. Livingston, "More 3-manifolds with multiple knot-surgery and branched-cover descriptions", Math. Proc. Cambridge Philos. Soc. 91:3 (1982), 473-475. MR 83j:57002

[Moser 1971] L. Moser, "Elementary surgery along a torus knot", Pacific J. Math. 38 (1971), 737745. MR 52 \#4287 Zbl 0202.54701

[Osoinach 1998] J. K. Osoinach, Jr., Manifolds obtained by Dehn surgery on infinitely many distinct knots in $S^{3}$, thesis, University of Texas at Austion, 1998.

[Osoinach 2006] J. K. Osoinach, Jr., "Manifolds obtained by surgery on an infinite number of knots

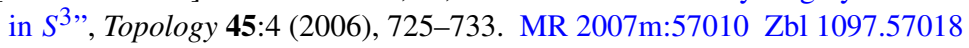

[Saito 2007] T. Saito, "Dehn surgery and (1, 1)-knots in lens spaces", Topology Appl. 154:7 (2007), 1502-1515. MR 2008f:57011 Zbl 1115.57005

[Saito 2008a] T. Saito, “The dual knots of doubly primitive knots”, Osaka J. Math. 45:2 (2008), 403-421. MR 2009e:57014 Zbl 1146.57012

[Saito 2008b] T. Saito, "Knots in lens spaces with the 3-sphere surgery", Algebr. Geom. Topol. 8:1 (2008), 53-79. MR 2009d:57014 Zbl 05236552

[Stallings 1978] J. R. Stallings, "Constructions of fibred knots and links", pp. 55-60 in Algebraic and geometric topology (Stanford, CA, 1976), vol. 2, edited by R. J. Milgram, Proc. Sympos. Pure Math. 32, Amer. Math. Soc., Providence, R.I., 1978. MR 80e:57004 Zbl 0394.57007

[Teragaito 1994] M. Teragaito, "Homology handles with multiple knot-surgery descriptions", Topology Appl. 56:3 (1994), 249-257. MR 95b:57013 Zbl 0811.57020

[Teragaito 2007] M. Teragaito, "A Seifert fibered manifold with infinitely many knot-surgery descriptions", Int. Math. Res. Not. 2007:9 (2007), Art. ID rnm 028. MR 2008g:57008 Zbl 1138.57012

[Wang 1989] S. C. Wang, "Cyclic surgery on knots", Proc. Amer. Math. Soc. 107:4 (1989), 10911094. MR 90e:57030 Zbl 0688.57008

[Wu 1990] Y. Q. Wu, “Cyclic surgery and satellite knots”, Topology Appl. 36:3 (1990), 205-208. MR 91k:57009 Zbl 0715.57002

[Yamada 2005] Y. Yamada, "Berge's knots in the fiber surfaces of genus one, lens space and framed links”, J. Knot Theory Ramifications 14:2 (2005), 177-188. MR 2005m:57016 Zbl 1086.57010

Received August 20, 2008. 


\section{Toshio SAITO}

DEPARTMENT OF MATHEMATICS

UNIVERSITY OF CALIFORNIA

SANTA BARBARA, CA 93106

UNITED STATES

tsaito@math.ucsb.edu

MASAKAZU TERAGAito

DEPARTMENT OF MATHEMATICS AND MATHEMATICS EdUCATION

HIROSHIMA UNIVERSITY

1-1-1 KAGAMIYAMA

HIGASHI-HIROSHIMA 739-8524

JAPAN

teragai@hiroshima-u.ac.jp 